Vittalle - Revista de Ciências da Saúde 29 n. 2 (2017) 79- 84
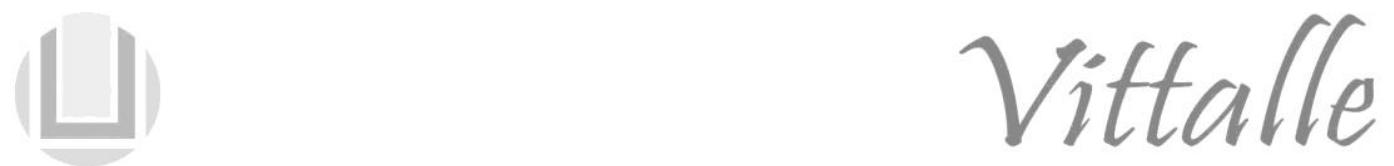

\title{
Questões éticas em dois consórcios de pesquisa
}

\author{
Renata Gomes Paulitsch ${ }^{\mathrm{a}^{*}}$, Adriana Vieira Camerinia ${ }^{\mathrm{a}}$, Samuel de Carvalho Dumith ${ }^{\mathrm{a}, \mathrm{b}}$, \\ Silvio Omar Macedo Prietch ${ }^{\mathrm{a}, \mathrm{b}}$, Susi Heliene Lauz Medeiros ${ }^{\mathrm{a}}$

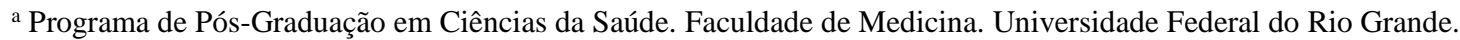 \\ Rio Grande, RS, Brasil. \\ ${ }^{\text {b }}$ Programa de Pós-Graduação em Saúde Pública. Faculdade de Medicina. Universidade Federal do Rio Grande. Rio \\ Grande, RS, Brasil.
}

Histórico do Artigo
Recebido em:
30/09/2016
Aceito em: 16/02/2017
Palavras-chave
Ética em Pesquisa
Estudos Transversais
Inquéritos
Demográficos.

Keywords Ethics in Research Cross-Sectional Studies Population Surveys

\begin{abstract}
RESUMO
A ética envolvendo estudos com seres humanos continua suscitando discussões no meio acadêmico. Mesmo em estudos que pressupõem mínimo risco, como os estudos observacionais, sempre há possibilidade de eventos não esperados. O objetivo foi discutir aspectos éticos envolvidos no desenvolvimento de dois consórcios de pesquisa: Saúde dos Universitários da Universidade Federal do Rio Grande e Saúde da População Riograndina. Para tanto, foram enfocadas considerações a respeito de estudos epidemiológicos, os dois consórcios de pesquisa, a segurança do paciente e situações imprevistas e eticamente relevantes. Podemos concluir que a atenção aos problemas éticos deve estar presente em pesquisas que envolvem seres humanos e que os participantes da pesquisa, tanto respondentes quanto aplicadores, podem estar envolvidos.

\section{ABSTRACT}

Ethics Studies involving with human beings continuous raising discussions not academic part. Even studies that assumes minimum risk as observational studies, there is always the possibility of unexpected events. The aim of this study was a reflection about the ethical issues in epidemiological studies involving human beings. Therefore used as bases two research consortium experiences. Were the studies "Health Riograndina Population" conducted in 2016 and "Health of the University of FURG" Held in 2015 in the city of Rio Grande, RS, Brazil. We can conclude that attention to ethical issues must be present in research that involve human beings and the subjects of the research, both respondents the applicators may be involved.
\end{abstract}

\section{Introdução}

Historicamente, desde que iniciaram as pesquisas científicas, abusos têm sido cometidos em nome da ciência. Experimentos científicos, sem o consentimento dos participantes, foram realizados durante as guerras mundiais e, em outras épocas, com grupos étnicos e economicamente vulneráveis. O Código de Nuremberg, de 1947, estabeleceu regras para o julgamento dos cientistas nazistas no pós-guerra; já a Declaração de Helsinque, em 1964, definiu os princípios gerais que deveriam ser seguidos pela pesquisa médica, sendo esses os primeiros documentos que criaram princípios éticos para as pesquisas em seres humanos $(1,2)$.

No Brasil, a Resolução Nº01, de 1988, do Conselho Nacional de Saúde (CNS), foi o primeiro passo para a regulamentação das pesquisas com seres humanos, estabelecendo normas para a pesquisa em saúde (3). Em 1996, a Resolução Nº196/96 foi publicada no Brasil, propondo diretrizes para pesquisas em seres humanos e, inclusive, estabelecendo que toda a pesquisa gera algum risco, ou seja, existe a probabilidade de que ocorra um evento desfavorável (4). Já, em 2012, o CNS aprovou novas diretrizes e normas de pesquisas envolvendo seres humanos, seguidas até os dias de hoje, por meio da

\footnotetext{
*Autor correspondente: renatapaulitsch@gmail.com (R. G. Paulitsch)
} 
Resolução Nº66/ 2012 (5)

Para realizar uma pesquisa que envolva seres humanos, é necessário conter um consentimento livre e esclarecido do participante (ou representante legal), que contemple quatro elementos: fornecimento de informações, compreensão, voluntariedade e consentimento (6). Os objetivos, justificativas e procedimentos que serão utilizados na pesquisa devem ser esclarecidos, utilizando uma linguagem clara e compreensível, ao nível de instrução do participante, assim como riscos e benefícios da pesquisa aos participantes ${ }^{5}$. É preciso, também, assegurar a voluntariedade em participar ou não da pesquisa, dando atenção especial a grupos vulneráveis ou que possam ser coagidos devido a funções profissionais ou institucionais (7). A autonomia plena do participante precisa ser estabelecida a todo momento, ou seja, o reconhecimento de que as pessoas podem tomar suas próprias decisões, devendo ser previamente informadas sobre procedimentos, seus riscos e consequências (7). Devem ser dadas garantias de anonimato e privacidade dos participantes. Assim, as pesquisas com seres humanos necessitam estar em consonância com os preceitos da bioética: autonomia, beneficência, não-maleficência e justiça (8). Nesse sentido, é essencial que sejam discutidos aspectos éticos que permeiam estudos transversais, já que inúmeros desafios se põem aos pesquisadores. Discutir as condutas e possibilidades dos mesmos torna-se fundamental para o crescimento das pesquisas.

O objetivo do presente artigo foi discutir aspectos éticos envolvidos no desenvolvimento de dois consórcios de pesquisa: Saúde dos Universitários da Universidade Federal do Rio Grande (FURG) e Saúde da População Riograndina.

\section{Desenvolvimento}

\section{CONSIDERAÇÕES A RESPEITO DE ESTUDOS EPIDEMIOLÓGICOS}

O Conselho de Organizações Internacionais de Ciências Médicas (CIOMS) propõe algumas diretrizes para estudos populacionais denominadas de "Pautas Internacionais para Avaliação Ética de Estudos Epidemiológicos” (9). Esse documento orienta que membros mais vulneráveis das populações não devem ser usados como participantes de pesquisas, por meio das quais toda a população será beneficiada, evitando uma distribuição injusta na seleção dos participantes (9). De preferência, os termos de consentimento devem ser obtidos individualmente e os resultados das pesquisas deveriam ser apresentados à comunidade ressaltando que os resultados de pesquisas epidemiológicas refletem situações populacionais e não de indivíduos. Outra questão que deve ser considerada é quanto a expectativas dos participantes em relação aos seus problemas de saúde. Segundo as "Pautas Internacionais para Avaliação Ética de Estudos Epidemiológicos", deve haver um sistema de referência aos serviços de saúde para os participantes que, no decorrer da pesquisa, necessitem de atendimento ${ }^{9}$. Como exemplo de estudos epidemiológicos, podemos relatar a experiência dos autores em dois estudos populacionais e as questões éticas envolvidas.

\section{CONSÓRCIO DE PESQUISA: SAÚDE DOS UNIVERSITÁRIOS DA FURG E SAÚDE DA POPULAÇÃO RIOGRANDINA}

Um "consórcio de pesquisa" trata-se de uma estratégia envolvendo a participação de vários pesquisadores em uma única coleta de dados. Para realização do consórcio, é necessária a construção de um único projeto (tema central), gerando um trabalho de campo comum a todos os envolvidos (10). 
Dois consórcios de pesquisa foram realizados por Programas de Pós-Graduação da FURG e serão apresentados a seguir.

O consórcio de pesquisa Saúde dos Universitários da FURG foi constituído por oito alunos de mestrado do Programa de Pós-Graduação em Saúde Pública da FURG. A partir dele, foi gerado um estudo transversal, incluindo os estudantes de graduação com 18 anos ou mais, matriculados no primeiro semestre de 2015 nos campi de Rio Grande. Foram coletadas informações por meio de questionários autoaplicados. As informações coletadas foram relacionadas a aspectos demográficos, socioeconômicos, comportamentais, sobre a vida universitária, saúde bucal, hábitos alimentares, vida sexual, uso de drogas lícitas e ilícitas, autoimagem e atividades físicas. A coleta de dados ocorreu de março a julho de 2015 pelos próprios mestrandos.

O consórcio de pesquisa Saúde da População Riograndina foi constituído por dez alunos de mestrado e doutorado dos Programas de Pós-Graduação em Ciências da Saúde e Saúde Pública da FURG. A partir dele, foi gerado um estudo transversal de base populacional com 1300 indivíduos com idade igual ou superior a 18 anos, residentes na zona urbana do município de Rio Grande, que teve por objetivo avaliar aspectos de sua saúde. Esse estudo obteve uma taxa de resposta de $91 \%$ com perdas de $9 \%$. As informações foram obtidas por meio de um questionário único, pré-codificado e padronizado, com predomínio de perguntas fechadas, testado em estudo piloto. Esse questionário coletou informações sobre características socioeconômicas, demográficas, comportamentais, alimentares e do ambiente físico. Dados a respeito do acesso e utilização de serviços de saúde, qualidade de vida, educação física e local para a prática desportiva e sobre saúde bucal foram também obtidos. A coleta de dados foi realizada por entrevistadoras treinadas e ocorreu de abril a julho de 2016.

Ambos os projetos de pesquisa foram submetidos ao Comitê de Ética em Pesquisa na Área da Saúde (CEPAS) da FURG, conforme os preceitos da Resolução 466/12. O consórcio com os universitários recebeu aprovação sob o parecer número 1.024.879, já o Saúde da população riograndina sob o número 1.473.344. Os princípios éticos foram resguardados para os entrevistados por meio dos seguintes itens: obtenção de Termo de Consentimento Livre e Esclarecido (TCLE); garantia do direito de não participação na pesquisa e sigilo acerca da identificação. Assim que ambas pesquisas foram encerradas, seus resultados foram divulgados às suas respectivas populações, com o objetivo de compartilhá-los com quem participou de forma voluntária.

Nossa experiência com tais estudos mostrou que a preocupação com a segurança dos participantes da pesquisa é essencial. E mesmo com todos os cuidados, ainda nos deparamos com questões imprevistas e eticamente relevantes.

\section{SEGURANÇA AOS PARTICIPANTES}

Risco, de acordo com a Resolução CNS N466/12, é a possibilidade de danos à dimensão física, psíquica, moral, intelectual, social, cultural ou espiritual do ser humano, em qualquer fase de uma pesquisa e dela decorrente (5).

Quaisquer modelos de pesquisa podem gerar risco aos participantes, tais como físicos, psicológicos, sociais ou econômicos. Sabe-se que estudos epidemiológicos apresentam um menor risco, mas podem gerar desconforto físico e psicológico. Ao aplicar questões que envolvem saúde mental e qualidade de vida, por exemplo, o indivíduo pode demonstrar sentimentos diversos (11).

No consórcio de pesquisa Saúde dos Universitários da FURG, foram utilizadas algumas estratégias para evitar constrangimento dos participantes. A presença de perguntas de foro íntimo envolvendo sexualidade e uso de drogas ilícitas levantou 
discussões na equipe de pesquisa quanto à necessidade de preservação da identidade dos participantes, inclusive os pesquisadores não puderam identificá-los. $\mathrm{O}$ anonimato nesse tipo de pesquisa é muito importante na adesão dos participantes. Como estratégia, foram usados questionários autoaplicados, cujo nome do participante não é conhecido e os instrumentos depositados pelos próprios participantes em urnas lacradas. Os alunos foram abordados em sala de aula, lhes tendo sido garantida toda a liberdade para deixarem o local ou desistirem de participar a qualquer momento.

No consórcio de pesquisa Saúde da População Riograndina, também foram realizadas algumas estratégias para assegurar a segurança dos participantes. As questões julgadas, pelos pesquisadores, mais sujeitas a causar desconforto foram alocadas em dois blocos (meio e final do questionário). Essa medida foi utilizada para que, no momento que as mesmas fossem feitas, o entrevistado já pudesse ter estabelecido uma relação com a entrevistadora. Em relação à aplicação dos questionários, as entrevistadoras foram orientadas a informar quando recebessem questionário de alguma pessoa conhecida para que fosse alocado para outra, evitando que as mesmas entrevistassem parentes e pessoas próximas.

Durante o treinamento das entrevistadoras, as questões éticas foram extensamente trabalhadas. Primeiramente, elas receberam orientações sobre a importância do TCLE e a sua função na pesquisa. Após, orientou-se sobre como manter e garantir o anonimato e a privacidade dos indivíduos participantes, sendo proibidos os comentários com colegas, família e conhecidos a respeito das respostas recebidas. Para assegurar esses pontos, foram realizados treinamentos, estudo piloto e acompanhamento das aplicações dos questionários pelos pesquisadores.

Os questionários preenchidos foram armazenados semanalmente em sala específica, dentro do Setor de Epidemiologia da FURG: digitados duplamente e armazenados em lotes. Os questionários não saíam do referido local, somente pessoal autorizado tinha acesso aos mesmos. É importante ressaltar que, na construção do banco de dados, os questionários não foram identificados pelo nome do indivíduo, mas por um número sequencial gerado para criação dos lotes.

\section{SITUAÇÕES IMPREVISTAS E ETICAMENTE RELEVANTES}

Conforme discutido anteriormente, as questões levantadas nos consórcios de pesquisa poderiam trazer desconforto aos entrevistados. No consórcio Saúde da População Riograndina, isso ocorreu em alguns momentos. Assim, descrevemos as decisões tomadas pelo grupo de pesquisadores. Cabe salientar que, semanalmente, o grupo reunia-se para decidir em conjunto as estratégias adotadas.

Do total de 1300 entrevistas, treze indivíduos solicitaram encaminhamento específico. Nove solicitaram encaminhamento a um dispositivo de saúde mental e quatro solicitaram encaminhamento para outros dispositivos de saúde (para atendimento odontológico, planejamento familiar, com fisioterapeuta e educador físico). Uma pessoa sentiu-se desconfortável e não conseguiu responder às questões sobre saúde mental e qualidade de vida, demonstrando instabilidade emocional (chorou).

Foram identificados 84 indivíduos com risco de suicídio, sendo necessário encaminhar para suporte. Esses participantes, mesmo não tendo solicitado encaminhamento, o receberam. Nesse momento, contrariou-se a confidencialidade da entrevista, sobrepondo o princípio da não maleficência, uma vez que esse antecede a autonomia (12).

No decorrer da coleta de dados, uma entrevistadora demonstrou desconforto emocional, algo não previsto pela equipe. Ao ter contato por mais de dois meses com as 
questões, a mesma iniciou um processo de reflexão acerca de seus sentimentos. Foi preciso traçar uma estratégia específica para essa situação, preservando o princípio da não maleficência, que deve ser empregado a todos os envolvidos em um consórcio de pesquisa e beneficência - agir em benefício do outro.

Para respeitar o direito de todos e não causar constrangimentos a quem não soubesse ler e/ou escrever, era disponibilizado, ao entrevistado, consentir sua participação por meio da coleta de sua digital. Dessa forma, mantinha-se a autonomia, que também compreende o auxílio ao indivíduo a superar seus sentimentos de dependência.

Tais situações, praticamente, não ocorreram com a pesquisa da Saúde dos Universitários da FURG, uma vez que os mesmos não precisaram externar nenhum sentimento ou situação pessoal. Aqueles estudantes que se sentiram desconfortáveis com as perguntas abandonaram o preenchimento do questionário. A equipe não se envolveu com as respostas dadas pelos participantes, assim, tornou-se praticamente inexistente o risco psicológico envolvido durante o trabalho de campo.

Em ambos consórcios, ocorreram mais de uma visita às salas de aula ou domicílios, a fim de reverter perdas ou recusas. Essa estratégia visava melhor acurácia dos dados coletados, porém levantou um questionamento entre os pesquisadores: qual o limiar entre a busca aos indivíduos para alcançar o rigor estatístico dos estudos epidemiológicos e o direito de não-resposta de cada um? Essa questão precisa ser pensada e refletida, pois não existe um limite explícito para as tentativas, mas, sim, um limite individual demonstrado.

\section{Considerações finais}

A importância dos estudos envolvendo seres humanos é inegável e tem contribuído muito para os avanços na área da saúde. Apesar de apresentar baixo risco aos participantes, os estudos epidemiológicos discutidos demonstram que a atenção aos problemas éticos deve estar sempre presente nos estudos com seres humanos. A atenção aos participantes da pesquisa deve ser estendida tanto aos respondentes quanto aos aplicadores, visto que ambos podem ser afetados. Muitas questões relacionadas com a ética na pesquisa podem surgir durante um trabalho de campo ou na aplicação do instrumento de pesquisa. Os limites dos pesquisadores devem estar sempre sendo discutidos dentro dos preceitos da bioética.

Conflito de interesses: Os autores declaram não haver conflito de interesses

\section{Referências}

1. de Nüremberg, Código. "Tribunal Internacional de Nüremberg." (1947).

2. Mundial, Associação Médica. "Declaração de Helsinque." Princípios éticos para a pesquisa em seres humanos. Jorge MR, tradutor. Helsinque; 1964.

3. Brasil. Conselho Nacional de Saúde. Resolução nº 001/1998. (DOU 14/06/1988: 10.713-10.718).

4. Brasil. Conselho Nacional de Saúde. Resolução n 196/96 sobre pesquisa envolvendo seres humanos. (DOU 16/10/96:21082-21085).

5. Brasil. Conselho Nacional de Saúde. Resolução nº 466 de 12/2012 (DOU 13/06/2013. Seção 1: 59).

6. English DC. Bioethics: a clinical guide for medical students. AORN Journal. 1994; 33-5.

7. Tegan C, Venancio PC, Marcondes FK, Rosalen PL. Autonomia e Vulnerabilidade do Sujeito da Pesquisa. Revista de Direito Sanitário. 2005; 6(1/2/3): 25-37.

8. Oliveira, PH, Filho RN. Bioética e Pesquisas em Seres Humanos. Revista da Faculdade de Direito da Universidade de São Paulo. 2006; 101: 1187-1227. 
9. Ciom, S. "International guidelines for ethical review of epidemiological studies." Ginebra; 1991.

10. Barros FC, Victora CG, Horta BL, Gigante DP. Metodologia do estudo da coorte de nascimentos de 1982 a 2004-5, Pelotas, RS. Rev Saúde Pública. 2008; 42 (2): 7-15.

11. Aquino EML, Vasconcellos-Silva PR, Coeli CM, Araújo MJ, Santos SM, Figueiredo RC, et al. Aspectos éticos em estudos longitudinais: O caso do ELSA-Brasil. Rev Saúde Pública. 2013; 47(12): 1926.

12. Loch, J.A. Princípios da Bioética. Uma Introdução à Bioética. Temas de Pediatria Nestlé. 2002(73): 12-19. 